DRAFT VERSION JANUARY 2, 2018

Preprint typeset using $\mathrm{LAT}_{\mathrm{E}} \mathrm{X}$ style emulateapj v. 11/26/04

\title{
DOMINANT NUCLEAR OUTFLOW DRIVING MECHANISMS IN POWERFUL RADIO GALAXIES ${ }^{1}$
}

\author{
Dan Batcheldor $^{2}$, Clive Tadhunter ${ }^{3}$, Johnna Holt $^{3}$, Raffaella Morganti $^{4}$, Christopher P. O’Dea $^{5}$, \\ DAVID J. AxON ${ }^{6}$ \& AnTON KoEKemoer ${ }^{7}$ \\ Draft version January 2, 2018
}

\begin{abstract}
In order to identify the dominant nuclear outflow mechanisms in Active Galactic Nuclei, we have undertaken deep, high resolution observations of two compact radio sources (PKS 1549-79 and PKS 1345+12) with the Advanced Camera for Surveys (ACS) aboard the Hubble Space Telescope. Not only are these targets known to have powerful emission line outflows, but they also contain all the potential drivers for the outflows: relativistic jets, quasar nuclei and starbursts. ACS allows the compact nature $\left(<0^{\prime \prime} .15\right)$ of these radio sources to be optically resolved for the first time. Through comparison with existing radio maps we have seen consistency in the nuclear position angles of both the optical emission line and radio data. There is no evidence for bi-conical emission line features on the large-scale and there is a divergance in the relative position angles of the optical and radio structure. This enables us to exclude starburst driven outflows. However, we are unable to clearly distinguish between radiative $\mathrm{AGN}$ wind driven outflows and outflows powered by relativistic radio jets. The small scale bi-conical features, indicative of such mechanisms could be below the resolution limit of ACS, especially if aligned close to the line of sight. In addition, there may be offsets between the radio and optical nuclei induced by heavy dust obscuration, nebular continuum or scattered light from the AGN.

Subject headings: quasars: emission lines - quasars: general - galaxies: active - galaxies: jets - galaxies: starburst - galaxies: individual (PKS 1549-79, PKS 1345+12)
\end{abstract}

\section{INTRODUCTION}

Gaseous nuclear outflows are known to be present in many Active Galactic Nuclei (AGN). Indeed, these high velocity phenomena have been noted in a broad range of objects, including Seyferts, starbursts, quasars and radio galaxies (Crenshaw et al.|2000; Krongold et al.|2003; Veilleux 2004; Morganti, Tadhunter \& Oosterloo 2005). In addition, it has been suggested that these outflows can (a) have a significant impact on the surrounding warm inter-stellar medium (ISM), which may in turn influence the evolution of the host galaxies, and can (b) limit the growth of the supermassive black holes and the rate of star formation (Silk \& Rees 1998; Wvithe \& Loeb 2003). As these factors link nuclear activity with the surrounding host galaxy and the process of galactic evolution, feedback effects associated with these outflows may be directly responsible for the observed tight relations between black hole mass and bulge properties (Ferrarese \& Ford 2005). However, there is a deficiency in our current understanding of AGN-induced feedback mechanisms. While the primary source for the large-scale $\mathrm{X}$-ray halos surrounding radio sources, in addition to the

\footnotetext{
${ }^{1}$ Based on observations made with the NASA/ESA Hubble Space Telescope obtained at the Space Telescope Science Institute, which is operated by the Association of Universities for Research in Astronomy, Incorporated, under NASA contract NAS 5-26555. These observations are associated with programme 10206.

2 Assistant Research Scientist, Center for Imaging Science, Rochester Institute of Technology, 54 Lomb Memorial Drive, Rochester, NY 14623

${ }^{3}$ Department of Physics \& Astronomy, University of Sheffield, Sheffield, S3 7RH, UK

${ }^{4}$ ASTRON, Postbus 2, 7900AA Dwingeloo, The Netherlands

${ }^{5}$ Department of Physics, Rochester Institute of Technology, Rochester, NY 14623

${ }^{6}$ Space Telescope Science Institute, 3700 San Martin Drive, Baltimore, MD 21218
}

kilo-parsec-scale radio structures, is seen to be the AGNinduced radio jet in many cases (e.g., Carilli et al. 1994; Nulsen et al. 2002; Fabian et al. 2003; Gallimore et al. 2006), the dominant driving device for these nuclear $(\leq 1$ $\mathrm{kpc}$ ) outflows is still unclear.

As radio-emitting jets can couple with the ISM more strongly than AGN photons, radio galaxies and radioloud quasars offer the chance to scrutinize the role of nuclear activity on the host galaxies of powerful AGN. In addition, since the light from bright quasar nuclei is extinguished along our line of sight by circumnuclear material, we can directly investigate the structure and spatial extent of the nuclear outflow regions. Gaseous outflows in powerful radio galaxies are also expected to be important for several reasons. Firstly, nuclear activity triggers, i.e., mergers or cooling flows (Heckman et al. 1986; Bremer, Fabian \& Crawford 1997), are likely to leave debris which is dissipated as the radio source evolves. Such nuclear debris has been implied by the greater number of absorption line systems in young, compact quasars than in quasars with extended, evolved radio morphologies (Baker et al. 2002). Secondly, NICMOS observations of Cygnus A have provided evidence of outflow induced despoliation in the form of hollowed out bi-conical structures (Tadhunter et al. 1999). Finally, it is likely that all the suggested drivers of the outflows are present to varying degree in radio galaxies.

There are several potential sources for the observed nuclear outflows: radiative winds from highly luminous quasar nuclei (Balsara \& Krolik 1993); starburst driven winds (Heckman. Armus \& Milev 1990); powerful relativistic jets (Bicknell. Dopita \& O'dea 1997); cloud interaction with expanding radio lobes (O'Dea et al.|2002). Each of these mechanisms will leave an imprint on the nuclear morphology of the host. 
TABLE 1

Summary of ObSERVATions

\begin{tabular}{|c|c|c|c|c|}
\hline Target & Instru. & Filter & $\underset{\AA}{\text { Central } \lambda}$ & $\begin{array}{l}\text { Exposures } \\
\text { No. } \mathrm{x} T(\mathrm{~s})\end{array}$ \\
\hline PKS 1549-79 & $\begin{array}{c}\text { WFC1 } \\
\text { HRC }\end{array}$ & $\begin{array}{c}\text { FR647M } \\
\text { FR459M } \\
\text { F550M }\end{array}$ & $\begin{array}{l}6798 \\
7560 \\
5234 \\
5580\end{array}$ & $\begin{array}{l}4 \times 250 \\
4 \times 250 \\
4 \times 730 \\
4 \times 700\end{array}$ \\
\hline PKS $1345+12$ & $\begin{array}{c}\text { WFC1 } \\
\text { HRC }\end{array}$ & $\begin{array}{c}\text { FR647M } \\
\text { FR459M } \\
\text { F550M }\end{array}$ & $\begin{array}{l}6618 \\
7361 \\
5093 \\
5580\end{array}$ & $\begin{array}{l}4 \times 200 \\
4 \times 200 \\
4 \times 640 \\
4 \times 620\end{array}$ \\
\hline
\end{tabular}

Note. - Exposures are giving in terms of total number, and length (in seconds), of each drizzle.

The main observational difference between AGN and starburst driven outflows will be in the size of the region from where the process originates. Starburst winds will have an origin which is roughly the size of the starburst region, i.e., $100-2000$ pc (Heckman, Armus \& Milev 1990), whereas the AGN wind driven outflows will have an origin which is inside the torus (e.g., Krolik \& Begelman 1986). Therefore, collimated outflows on scales of $<100 \mathrm{pc}$ would indicate that the outflow is AGN driven. A secondary diagnostic is the alignment of the outflow with the minor axis of the galaxy. Since starburst driven winds are collimated by the galaxy pressure gradient, they should align with the minor axis. However, the torus that defines the AGN driven outflows does not necessarily have to be aligned with the galaxy minor axis as Seyfert radio sources show a roughly random distribution of orientation with respect to the minor axis (e.g., Kinney et al. 2000).

In jet driven outflows the emission line structure will have similar features and scales to the radio structure. However, in lobe driven outflows, where the expanding radio lobe bow shock runs over ambient clouds giving them a kick outward, the cloud velocities are much smaller than the radio source bow shock expansion velocity. Therefore, the outflow region will be smaller than the total radio source extent.

The following analysis is designed to determine the dominant mechanism driving nuclear outflows through the study of two compact radio sources. For an extended review of such objects see O'Dea (1998). Firstly, the nearby compact flat-spectrum radio source PKS 1549-79 ( $\mathrm{z}=0.152)$. Secondly, the nearby Gigahertz Peaked Spectrum (GPS) radio source PKS 1345+12 $(\mathrm{z}=0.122)$. Both these sources are hosted by ultraluminous infra-red galaxies (URLIGS), with on-going star formation, and are well known powerful radio galaxies in which outflows have been unequivocally detected in the near nuclear narrow-line region, i.e., Holt. Tadhunter \& Morganti (2003, hereafter HTM03) and Holt et al. (2006, hereafter H06). In addition, these previous spectroscopic observations indicate that the [OIII] emission in the nuclear regions is dominated by broad, outflowing emission line gas. Therefore, despite accepting all [OIII] emission from the nuclear regions, suitable filters will avoid any ambiguities associ- ated with possible contamination by non-outflowing, narrower emission line components in the nuclear regions.

Important information on the outflow driving mechanism could be gained through further consideration of the internal nuclear kinematics and also from studying the possible ionization mechanisms. The relative velocities and FWHM of the kinematical components in both PKS 1549-79 and PKS $1345+12$ are consistent with shocks, however, the same cannot be said about the ionization mechanism. In the case of PKS 1549-79, the line ratios are consistent with AGN photoionization; there is no evidence for shock ionization. In PKS $1345+12$, the nature of the outflow components change between different lines making it impossible to investigate the ionisation mechanisms using the standard line ratios and diagnostic diagrams.

We introduce high resolution emission line maps obtained with the Advanced Camera for Surveys (ACS) aboard the Hubble Space Telescope $(H S T)$. The superior spatial resolution of ACS allows the compact nature of these sources $\left(\sim 0^{\prime \prime} .15\right)$ to be resolved for the first time. These new data make it possible to distinguish the main outflow driving mechanism through morphological comparison with existing radio data.

In $\S 2$ we detail the $H S T$ observations and the data reduction steps. The new ACS data are presented in $\S 3$ and $\S$ 4. These observations, in conjunction with VLBI data, allow us to attempt to identify the mechanisms dominating the nuclear outflows in $\S$ 5. Our findings are discussed in $\S 6$ and concluded in $\S[$.

\section{OBSERVATIONS AND DATA REDUCTION}

PKS 1549-79 and PKS 1345+12 were observed by ACS on the $4^{\text {th }}$ and $5^{\text {th }}$ August 2004 as part of Cycle 13 \#10206 PI: Tadhunter. Two exposures of PKS 1549-79, each of $4 \times 250$ s, were made using chip 1 of the Wide Field Channel (WFC1, pixel scale $\approx 0$.'05 - the spatial scale at the distance of these targets is $\sim 3 \mathrm{kpc} / \operatorname{arcsec})^{7}$. The IRAMP (FR647M, $\delta \lambda=9 \%$ ) ramp filter was adjusted to cover two central wavelengths, $7560 \AA$ for the $\mathrm{H} \alpha$ emission line and $6798 \AA$ for the continuum map. To avoid overfilling the HST storage buffer only a $1024 \times 1024$ subarray of the WFC1 was read out. Two more exposures of PKS 1549-79 were also made using the High Resolution Channel (HRC, pixel scale $\approx 0.027$ ). The first focused on the [OIII] emission line (F550M, $\delta \lambda=547 \AA, 4 \mathrm{x} 700 \mathrm{~s})$ and the second focused on the continuum (FR459M $5234 \AA, \delta \lambda=9 \%, 4 \mathrm{x} 730 \mathrm{~s})$. This strategy was repeated for PKS $1345+12$ where the central wavelength of the FR647M ramp filter was adjusted to $7361 \AA$ and $6618 \AA$ (4x200s each), and $5093 \AA$ for the FR459M ramp filter (4x640s). A summary of these observations is presented in Table 1

The data were passed through the ACS reduction pipeline, using the latest reference files, in order to remove instrumental residuals. Cosmic-ray removal was facilitated through the use of the Multi-drizzle script (Koekemoer et al. 2002). Detailed examinations of the distortion corrected data were performed, given that only subsets of the data were read out. The ellipticity of isophotes fitted to 10 stellar objects across each field

7 We adopt a value of $\mathrm{H}_{0}=70 \mathrm{~km} \mathrm{~s}^{-1} \mathrm{Mpc}^{-1}$ throughout 

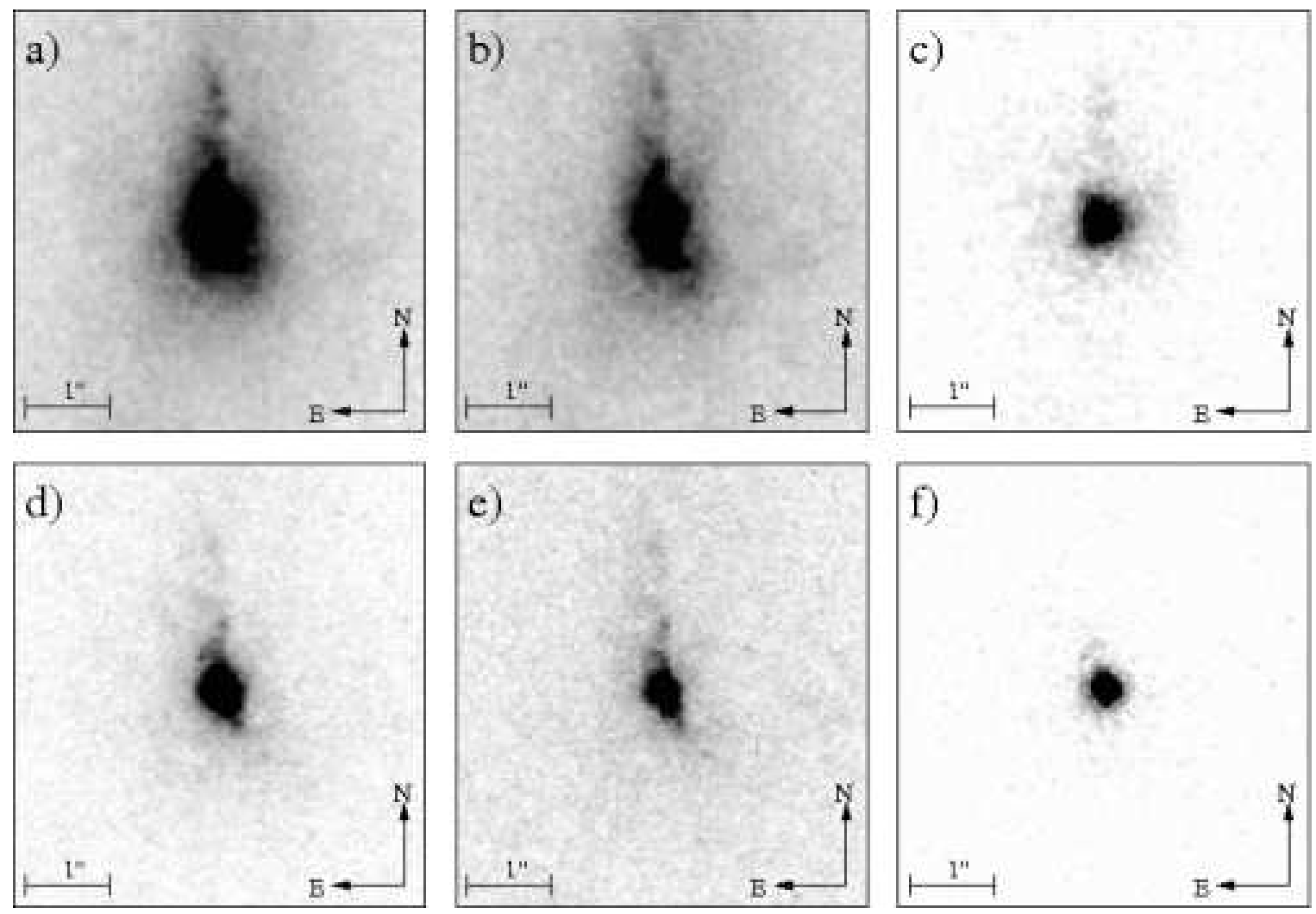

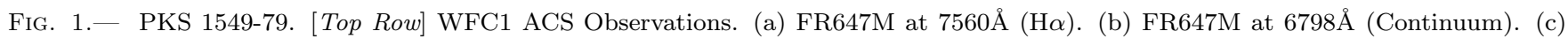

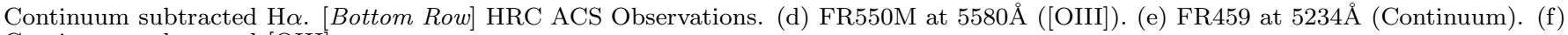
Continuum subtracted [OIII].

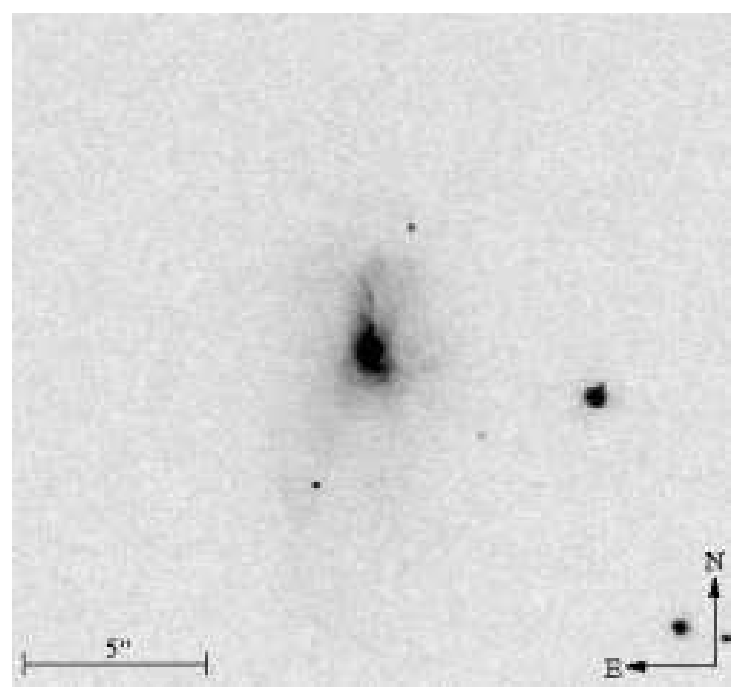

FIG. 2.- PKS 1549-79. The larger structure (FR647M at $6798 \AA$ ) showing extended diffuse with potential star clusters and foreground stars.

never exceeded 0.08 and averaged 0.03. As a final step, the on and off line images were scaled and subtracted from each other in order to produce emission line maps.

\section{PKS 1549-79}

The radio source PKS 1549-79 has had much previous work carried out on it. A brief outline of its main properties will be given before the new data are presented. Readers interested in more thorough reviews are directed toward Morganti et al. (2001), Tadhunter et al.
(2001) and H06.

VLBI observations reveal PK1549-79 to have a relatively small ( $\sim 150$ mas) one-sided distorted jet structure bending through $60^{\circ}$ (King 1994). Lower resolution maps show no evidence of large scale structures. The radio jet has a steep radio spectrum and originates from an unresolved flat spectrum radio core. Although the jet is likely close to our line of sight this source shows no evidence for broad emission-line features, contrary to the standard orientation based unification schemes. A model describing a cocooned (obscured) quasar, where a young radio jet is carving a path through a dense nuclear region, has been proposed by Tadhunter et al. (2001). A narrow HI absorption line gives a redshift of 0.152 (Morganti et al. 2001, H06). Prestage \& Peacock (1983) were first to associate the radio source with what has been classified as an 18.5(V) magnitude Seyfert 2.

Signs of star formation are provided by abnormally strong far-infrared (FIR) emissions, i.e., ULIRG, and an optical continuum that is dominated by a population of early type stars (Roy \& Norris 1997; Dickson 1997). Based on spectral synthesis modeling, H06 have shown this young stellar population to account for 1-30\% of the total stellar mass, and be between 50 and 250 Myrs old. In addition, the modeling of $\mathrm{H} 06$ has shown that the reddened quasar, which has been directly detected in the Kband (Bellamy et al. 2003), makes a small but significant contribution to the continuum around the wavelength of $\mathrm{H} \alpha$. This has also been suggested by the detection of a very broad component to the $\mathrm{H} \alpha$. Further spectral analyses (Tadhunter et al. 2001, H06) have shown the out- 

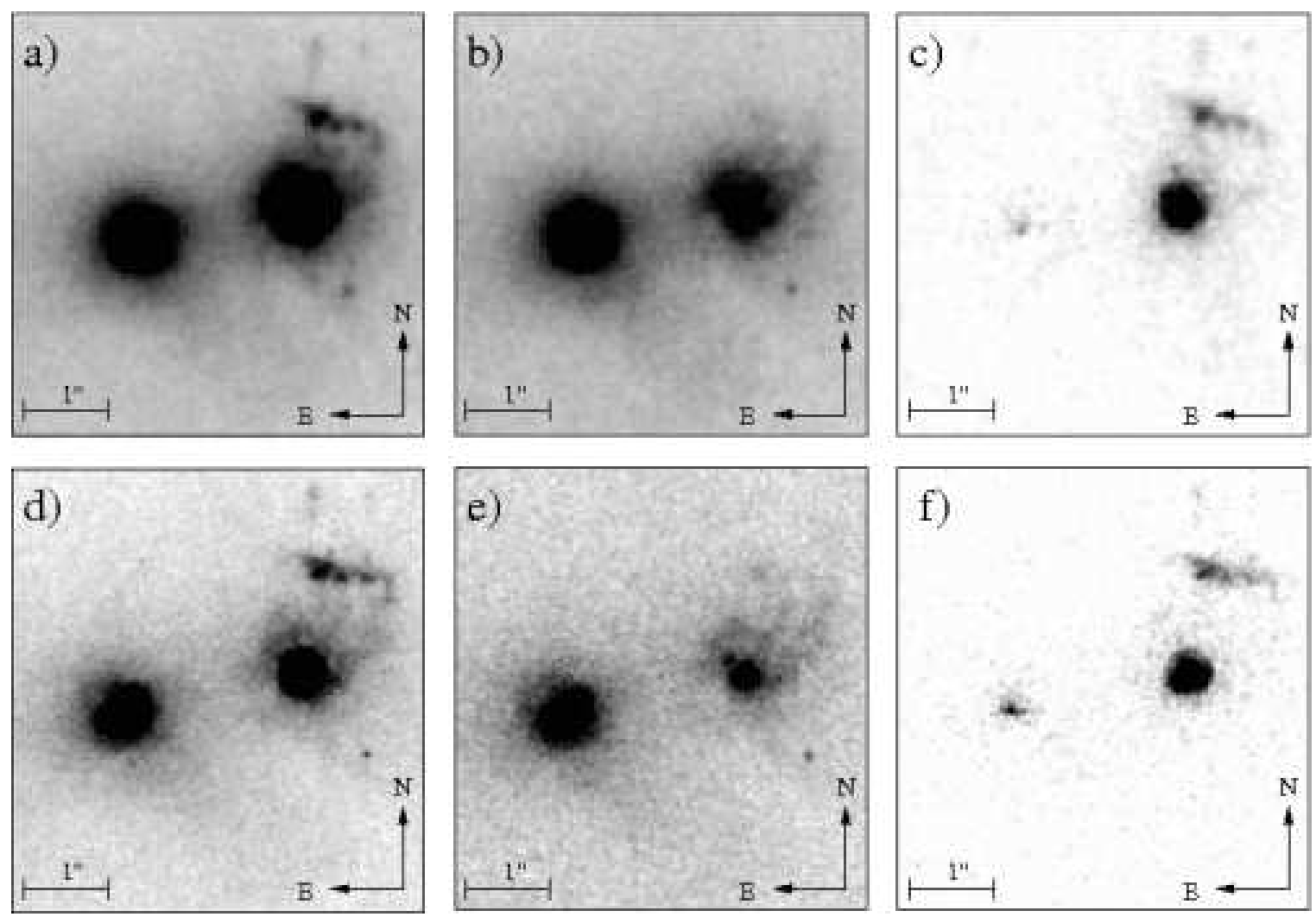

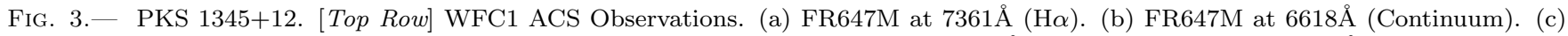

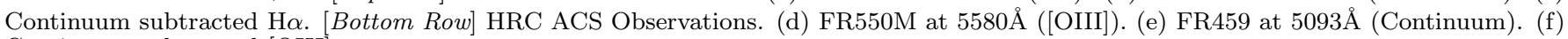
Continuum subtracted [OIII].

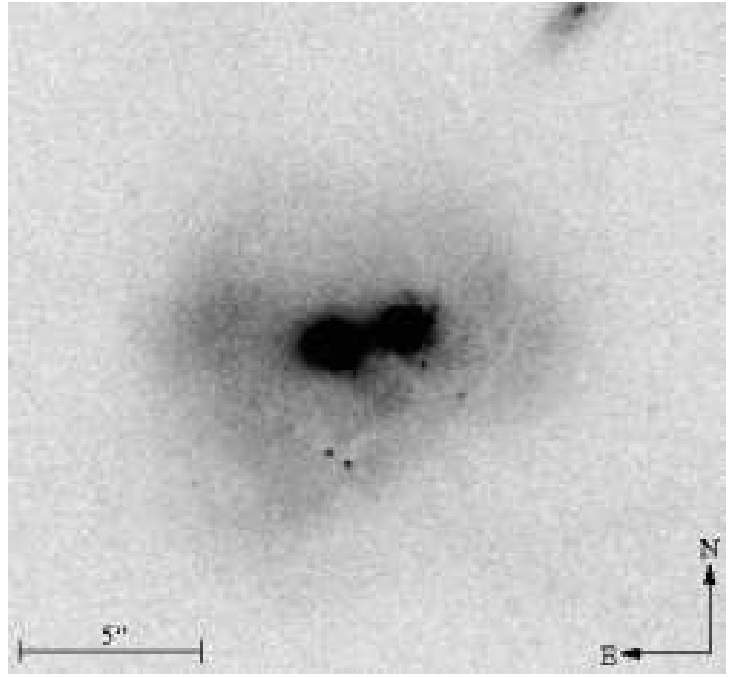

FIG. 4.- PKS 1345+12: The larger scale structure (FR647M at $6618 \AA$ ) showing the distorted morphology.

flow to have an extremely broad high ionization [OIII] line (FWHM 1280 $\mathrm{km} \mathrm{s}^{-1}$ ), and a lower ionization [OII] line $\left(\mathrm{FWHM} \approx 380 \mathrm{~km} \mathrm{~s}^{-1}\right)$. The outflow, which is blueshifted by $680 \mathrm{~km} \mathrm{~s}^{-1}$ with respect to the rest frame, is detected in all of the ionization lines. However, the outflow is strongest (and dominated by) the high ionization lines. In comparison, the low ionisation lines are dominated by quiescent gas.

In summary, H06, using ground-based optical, infrared (IR) and radio observations, have confirmed that
TABLE 2

Astrometric Details

\begin{tabular}{|c|c|c|c|}
\hline & \multirow[t]{2}{*}{ PKS 1549-79 } & \multicolumn{2}{|c|}{ PKS $1345+12$} \\
\hline & & (East) & (West) \\
\hline$H S T$ RA: & $15^{\mathrm{h}} 56^{\mathrm{m}} 58^{\mathrm{s}} .96$ & $13^{\mathrm{h}} 47^{\mathrm{m}} 33.51$ & $13^{\mathrm{h}} 47^{\mathrm{m}} 33^{\mathrm{s}} .37$ \\
\hline$H S T$ DEC: & $-79^{\circ} 14^{\prime} 04^{\prime \prime} 16$ & $+12^{\circ} 17^{\prime} 22^{\prime \prime} .99$ & $+12^{\circ} 17^{\prime} 23^{\prime \prime} .44$ \\
\hline Radio RA: & $15^{\mathrm{h}} 56^{\mathrm{m}} 58^{\mathrm{s}} .87^{1}$ & \multicolumn{2}{|c|}{$15^{\mathrm{h}} 56^{\mathrm{m}} 58^{\mathrm{s}} 87^{2}$} \\
\hline Radio DEC: & $-79^{\circ} 14^{\prime} 04^{\prime \prime} 28^{1}$ & \multicolumn{2}{|c|}{$-79^{\circ} 14^{\prime} 04^{\prime \prime} 28^{2}$} \\
\hline$\Delta \mathrm{RA}:$ & $1^{\prime \prime} 35$ & $2^{\prime \prime} 25$ & $0 . \prime 15$ \\
\hline$\triangle \mathrm{DEC}:$ & $0 . \prime 12$ & $1^{\prime \prime} 27$ & $0 !{ }^{\prime \prime} 82$ \\
\hline
\end{tabular}

Note. - All co-ordinates are in J2000. (1) Radio data from Johnston et al. (1995). (2) Radio data from Stanghellini et al. (2001).

PK1549-79 constitutes a young luminous narrow-line (obscured) quasar, the activity of which has been triggered by a gas rich major merger.

In Figure 1 we present the new ACS data for PKS 1549-79. In both the $\mathrm{H} \alpha$ and [OIII] line and continuum images we find a prominent nucleus with an offshoot extending to the north. The radio and HST astrometry is listed in Table 2, The offshoot, which is significantly less prominent in the [OIII] emission, appears to have several distinct components, the furthest of which lies along a position angle (PA) of 0 and at a distance of $1^{\prime \prime} 6(\sim 5$ kpc) from the nucleus. The sharpness of the features in this northern offshoot, and the block-like appearance of the isophotes to the south-east $\left(\mathrm{PA} \approx 15^{\circ}\right)$ of the nucleus provide evidence for a dust lane with a PA similar to the 
PA of the nuclear emission line and continuum structures (e.g. Figure 1d).

On the large scale (Figure 2) we can see extremely faint, diffuse emission that extends for $\sim 6 . .5(20 \mathrm{kpc})$ in the east-west direction. The north-south extension, as noted by Prestage \& Peacock (1983), can be seen. This Figure shares similar features to the deep Very Large Telescope Gunn $r$ image of H06 (their Figure 1). The authors note that the north-south extended features appear to contain knots of emission. Here we can see point-like sources in similar positions to these knots, which could be foreground stars but may also be super star clusters associated with the tidal tails of PKS1549-79.

\section{PKS $1345+12$}

The radio source PKS $1345+12$ has also had much previous work carried out on it. Again, a brief outline of its main properties will be given before the new data are presented. Readers interested in a more thorough review are directed toward HTM03.

PKS 1345+12 (4C 12.50) is one of the closest GPS sources. This allows the compact double jet and core structure, noted from VLBI $(2 \mathrm{~cm})$ and VLBA $(6 \mathrm{~cm})$ radio observations, to present proportions of $\sim 00^{\prime \prime} 15$. The more prominent S-shaped jet component extends to the south-east before bending and expanding into a diffuse lobe. Protruding to the north-west of the core (the core being identified from the relative flatness of the radio spectrum) weak radio emission is detected. On larger scales, diffuse radio emission is detected extending 35".0 ( $\sim 80 \mathrm{kpc}$ ) to the north, and $25^{\prime \prime} 0(\sim 55 \mathrm{kpc})$ to the south (Stanghellini et al. 2005).

Optically, PKS 1345+12 shows a complex morphology characterized by two nuclei, the western most of which, identified as a $17.5(\mathrm{~V})$ magnitude elliptical, possessing an extended curved tail. Previous HST studies, which are unable to resolve structure down to the scale of the radio morphology, have shown the western nucleus to be associated with the radio source Axon et al. 2000). From the ACS data presented here we find optical - radio offsets (Table 2) similar to those found by Axon et al. (2000), which confirms the western nucleus as the host of the radio source. The double nucleus and distorted appearance of PKS 1345+12 shows that a merger event is taking place. As with the other source in this study, PKS $1345+12$ shows a young stellar population (Tadhunter et al. 2005), a FIR excess (ULIRG) and nearUV emission (Evans et al. 1999, Labiano et al., in prep), indicating the presence of considerable star formation. Using optical spectroscopy, HTM03 have observed 3 distinct nuclear kinematical components in PKS 1345+12, the narrowest of which $\left(\mathrm{FWHM} \approx 340 \mathrm{~km} \mathrm{~s}^{-1}\right)$ is interpreted as the systemic velocity. The two other components, designated "intermediate" and "broad", show FWHM of $\sim 1250 \mathrm{~km} \mathrm{~s}^{-1}$ and $\sim 1950 \mathrm{~km} \mathrm{~s}^{-1}$ respectively, with corresponding blue-shifts of $\sim 400 \mathrm{~km} \mathrm{~s}^{-1}$ and $\sim 1980 \mathrm{~km} \mathrm{~s}^{-1}$ with respect to the rest frame. Due to the reddening observed in each component, it is proposed that the broad component originates from the inner most regions closest to the obscured quasar, whilst the narrow component represents a quiescent halo.

In Figure 3 we present the new ACS data for PKS $1345+12$. In both the $\mathrm{H} \alpha$ and [OIII] line and continuum images we find the two separate nuclei clearly vis- ible within an extended diffuse envelope. The $2 . \prime 0(\sim 5$ kpc) separation of the two nuclei measured here is $\sim 00^{\prime \prime} 1$ greater than the separation reported by Heckman et al. (1986) and Gilmore \& Shaw (1986). The continuum subtracted emission line images (Figures $3 \mathrm{r}$ c and $\mathrm{f}$ ) show that an insignificant amount of line emission emanates from the eastern component when compared to the western nucleus. To the north-west of this western nucleus there is an extended emission line filament (e.g., Figures $3 \mathrm{a}$, $\mathrm{c}, \mathrm{d}$ and f). Immediately north-east of the main nuclear structure there is a separate island of [OIII] emission (see Figure 3 $\mathrm{k}$ ) and a "mushroom" like morphology to the $\mathrm{H} \alpha$ continuum (see Figure 3b); this is clear evidence of a dust lane running approximately south-east to north-west. On the larger scale we can again compare this data to the findings of Heckman et al. (1986). Figure 4 highlights the distorted morphology and hints at some of the south-west curved tail extensions mentioned by Heckman et al. (1986). We also see that the halo of PKS 1345+12 contains similar point like sources (to the south of the eastern nucleus) to the foreground stars or possible star cluster seen around PKS 1549-79. Further study has shown these sources to be super star cluster with young stellar populations (Rodriguez Zaurin et al. 2006).

\section{INVESTIGATING THE DOMINANT MECHANISM}

In this section we closely examine the inner structures of the observed nuclei and determine the most likely position of the AGN in the ACS emission line images (and therefore the likely position of the radio cores). Biconical structures will suggest that the outflows are wind driven, whereas structures similar to the radio morphologies will suggest that the outflows are driven by the radio jets. However, first we examine the relative astrometry in the ACS data in order to estimate the uncertainties in derived positions.

\subsection{Relative Astrometry and Alignments}

There are two elements that could contribute to differences between the spatial offsets of objects measured on the WFC1 and HRC frames: the errors in measuring the positions themselves, i.e., true positions as related to measured positions, and the residual errors in the correction of the spatial distortion by the pipeline reduction. By quantifying these errors we can estimate the astrometric uncertainties present in the ACS images. An estimate of the residual distortion error can be gained from the relative positions of stellar like objects present in both the WFC1 and HRC data. The errors in measuring the positions themselves can be minimized by averaging the differences across as many objects as possible. We identified 7 stellar like objects common to the PKS 1549-79 data (the PKS 1345+12 field is relatively empty) and centroided their positions. Each stellar object was then paired with the remaining six objects, and the offset between the pairs were recorded (21 total offsets). The dispersion in the relative offsets between these objects in the WFC1 and HRC images, which we adopt as our astrometric uncertainty, was found to be $\sim 0^{\prime \prime} 03$.

In addition to the astrometric uncertainties, the obscured nature of the central engines can be an added source of error for the relative alignments of the AGN 


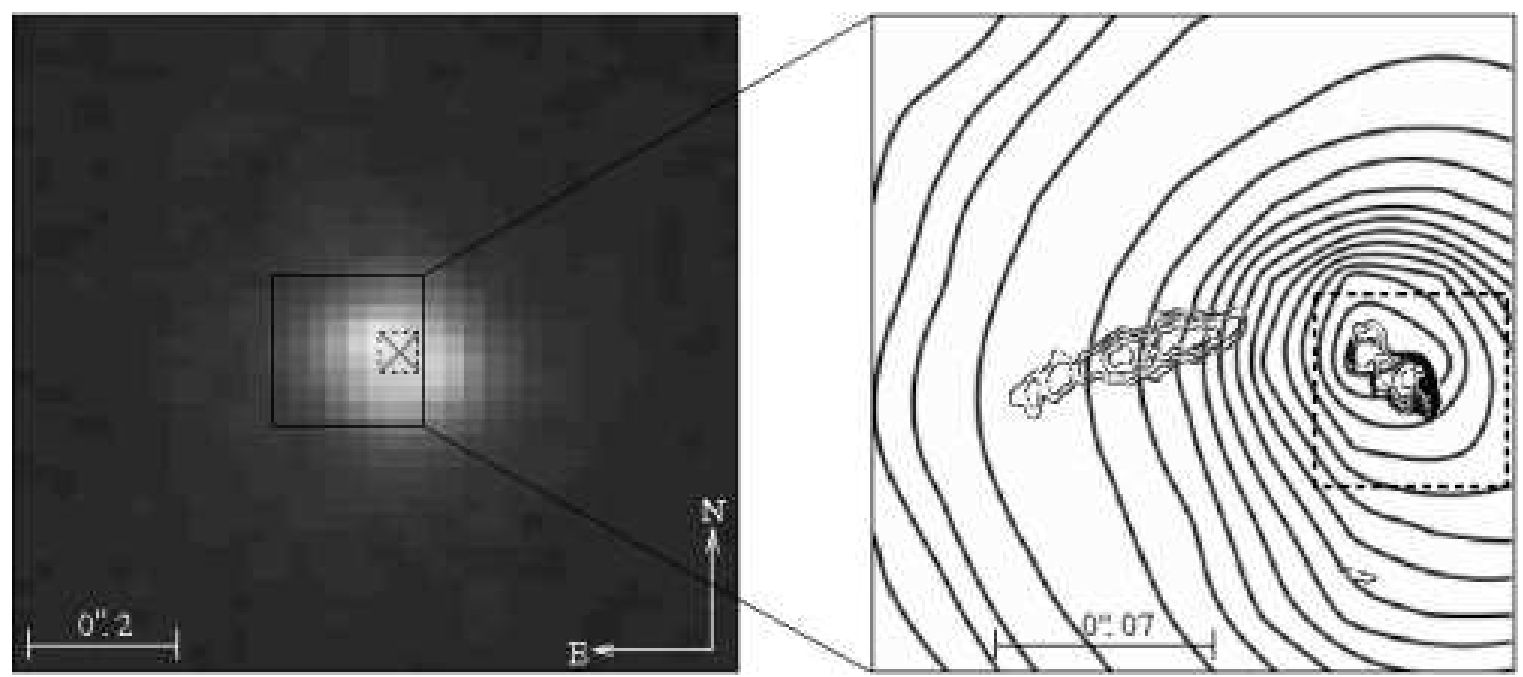

FIG. 5.- PKS 1549-79: Comparing the ACS data with the radio morphology. On the left is the HRC continuum subtracted [OIII] emission line map with a logarithmic stretch showing the very high surface brightness features. An "X" marks the position of the H $\alpha$ core and dotted lines show the estimated astrometric error box. On the right we see an expanded view of the [OIII] emission (smoothed thick contours) overlaid with the $2.3 \mathrm{GHz}$ radio map (thin contours at 10, 20, 40, 80, 160, 320, 640 and $1280 \mathrm{mJy} \mathrm{Beam}^{-1}$ ).

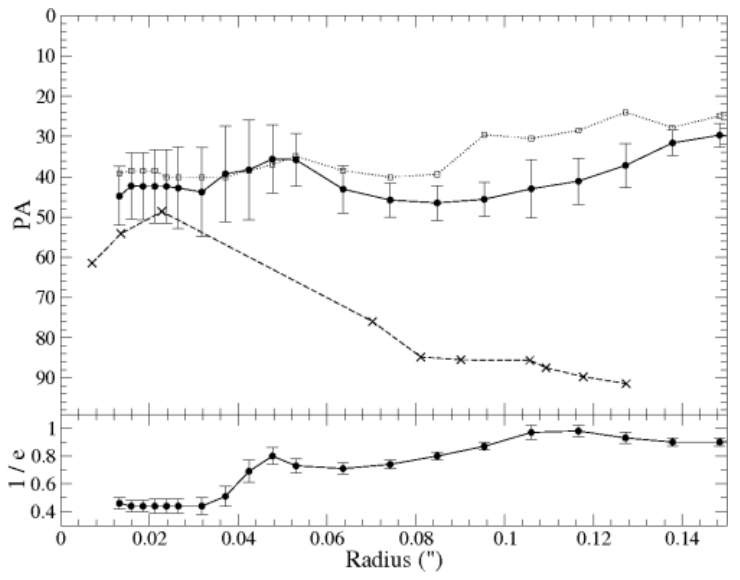

FIG. 6.- PKS 1549-79: Comparing continuum subtracted [OIII] and radio position angles. In the top panel, positions of knots in the radio emission, with respect to the radio core, are plotted as crosses and joined with a dashed line, while the PAs of ellipses fitted to the [OIII] continuum subtracted emission are joined with a solid line. The PAs of the [OIII] continuum are plotted with open squares and joined with a dotted line, the errors are omitted for clarity but are at the same level as for the [OIII] emission. The lower panel shows the ellipticities of the [OIII] continuum subtracted emission line data.

and radio cores; the measured peak in emission line flux may not correspond to the position of the AGN. In the following two sections we will attempt to minimize this effect, however, for this process to be a significant source of error it must act at a distance of $>00^{\prime \prime} 03(100 \mathrm{pc})$ from the estimated position of the central AGN.

\subsection{PKS 1549-79}

The very central region (1".0) of PKS 1549-79 ([OIII] emission line) is presented in Figure 5(a). We see a relatively high surface brightness concentration to the northeast, with a lower surface brightness fan of emission extending toward the south-west. Based on existing optical and near-IR spectra we do not expect to be able to detect the reddened AGN in the HRC [OIII] images. However, we know from the modeling presented by H06 that the quasar nucleus should be detected in the $\mathrm{H} \alpha$ continuum image. Therefore, we have assumed that the centroid of the concentration of pixels at the center of the WFC1 $\mathrm{H} \alpha$ continuum image gives the true quasar position relative to the reference stars common to both the WFC1 and HRC fields. When translated to the HRC images the WFC1 position is found to lie at the point marked with an " $\mathrm{X}$ " in Figure 5(a). It is with this point that we align the radio core. The estimated astrometric uncertainty is outlined with dotted lines $\left( \pm 00^{\prime \prime} 03\right)$.

The radio map in panel (b) of Figure 5 (thin contours) is that presented by H06. The data were collected in 1988 at $\sim 3 \mathrm{GHz}$ using a the Southern Hemisphere VLBI Experiment, SHEVE (Preston et al. 1984). The beam size is $7.3 \times 2.7$ mas at a PA of $4.7^{\circ}$. The western-most knot shows a flat spectrum across $2.3-8.4 \mathrm{GHz}$ and is considered the core. The $\mathrm{H} \alpha$ AGN position has subsequently been aligned with this. A steep spectrum jet is seen to extend to the east at a PA of $\sim 90^{\circ}$. The jet bends through approximately $60^{\circ}$, indicative of orientation change, and exhibits a gap which may be attributed to cyclic emission processes. H06 estimate an upper limit of $i<55^{\circ}$ for the inclination, with respect to the line of sight, for this jet.

Figure 5(b) also demonstrates the comparative morphologies between the radio structure (thin contours) and the [OIII] emission (thick lines). The emission line map shows no evidence for bi-conical features. Ellipse fitting to the [OIII] data is presented in Figure 6, where the PAs of knots along the jet, with respect to the radio core, are also plotted. We see excellent agreement between the [OIII] continuum and [OIII] emission line PAs. Similarity in the emission line and continuum morphology could be attributed to dust obscuration, scattered AGN light (Tadhunter et al. 1992), nebular continuum (Dickson et al. 1995), or a mixture of all three. In addition to the optical emission-continuum alignment, there is an overlap in the very inner regions between the radio structure and the [OIII] emission. However, this radiooptical agreement deteriorates as the jet curves around. The jet also extends past the highest surface brightness region of the [OIII] emission. 


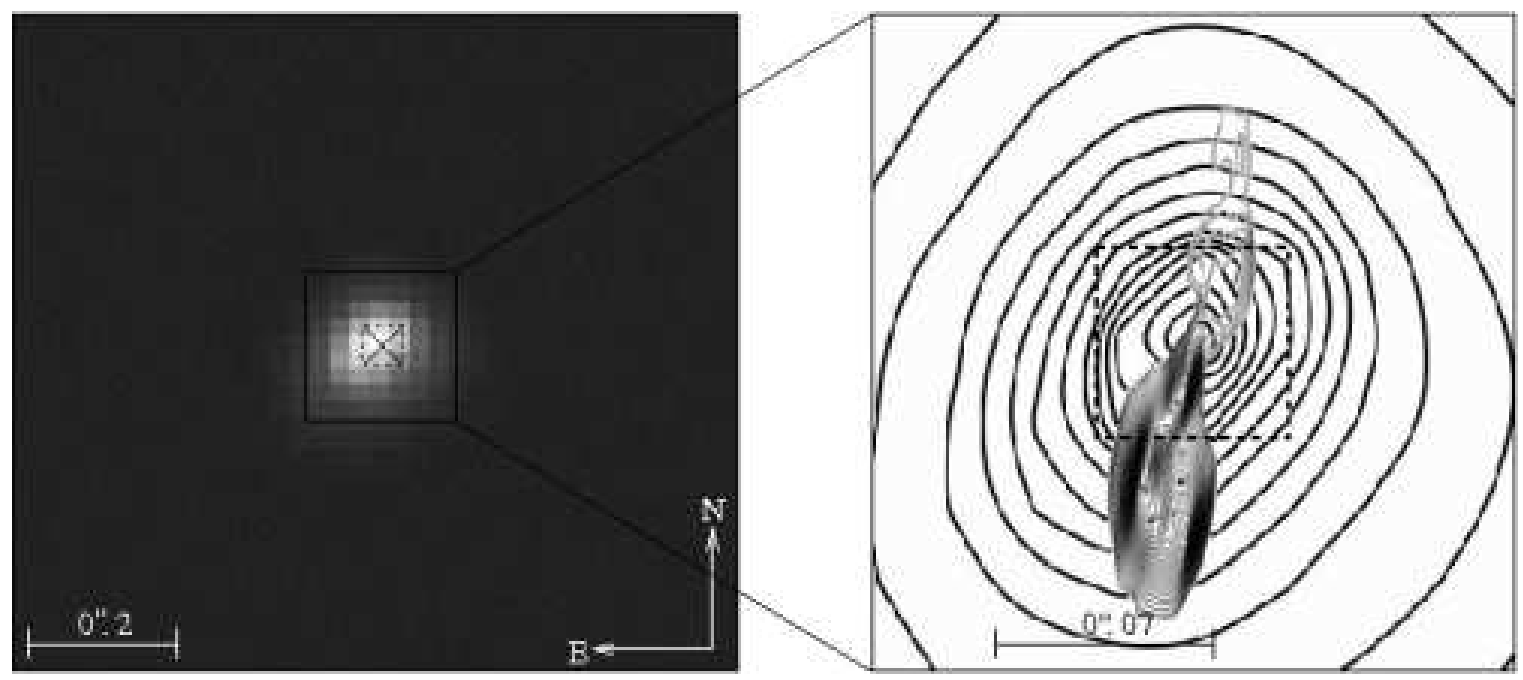

FIG. 7.- PKS 1345+12: Comparing the ACS data with the radio morphology. On the left is the HRC continuum subtracted [OIII] emission line map with a logarithmic stretch showing the very high surface brightness features. An "X" marks the position of the NIR core and dotted lines show the estimated astrometric error box. On the right we see an expanded view of the [OIII] emission (smoothed thick contours) overlaid with the $1.3 \mathrm{GHz}$ radio map (thin contours at $7,10,16,25,37,42 \ldots 766 \mathrm{mJy} \mathrm{Beam}^{-1}$ ).

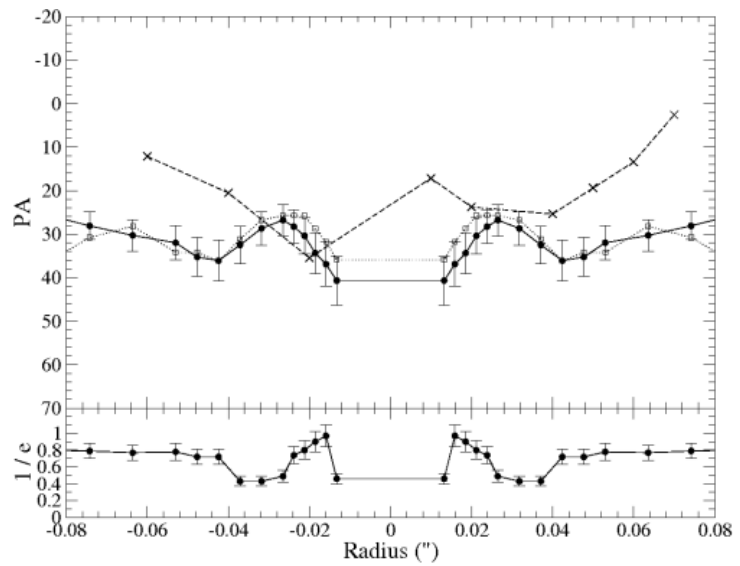

FIG. 8.- PKS 1345+12: Comparing continuum subtracted [OIII], [OIII] continuum and radio position angles. As Figure 6. but for PKS $1345+12$.

\section{3. $P K S 1345+12$}

The very central region of the western nucleus in PKS 1345+12 ([OIII] emission line) is presented in Figure 7(a). A north-west to south-east elongation is clearly apparent. As in the case of PKS 1549-79, we do not expect to be able to directly detect the AGN in the [OIII] images, nor at optical wavelengths. However, we can assume that the quasar nucleus will be detected in the $\mathrm{K}$ band. Therefore, we have retrieved a NICMOS F222M image for this source from the HST $\operatorname{archive}^{8}$. It was taken as part of programme \#7219 PI: Scoville, the finer details of which are presented in Scoville et al. (2000). By determining the relative offset of the eastern nucleus with that of the western nucleus in both the emission line images and the NIR, we can place a better constraint on the position of the radio core. Following this we have indicated in Figure 7(a) the position of the NIR peak with respect to the eastern nucleus on the HRC emission line map. It is here we assume the radio core to fall.

The radio map in panel (b) of Figure [7 (thin con- tours) is a $1.3 \mathrm{GHz}$ map obtained from a global VLBI (HI) experiment (Morganti et al. 2004) overlaid onto the smoothed contours of the HRC emission line map (thick lines). The radio data were collected in 2001 where the beam size was $6.6 \times 1.2$ mas at a $\mathrm{PA}$ of $-11^{\circ}$. The radio core has been identified at $5 \mathrm{GHz}$ by Stanghellini et al. (1997) from the relatively flat spectrum. However, similar to the findings of Xiang et al. (2002), this core is not clearly defined in the $1.3 \mathrm{GHz}$ map, and is likely self absorbed. Therefore, in order to register the $1.3 \mathrm{GHz}$ map (which shows more extended, lower surface brightness features than the $5 \mathrm{GHz}$ map) against the optical emission line images, we have aligned the $5 \mathrm{GHz}$ map with the $1.3 \mathrm{GHz}$ map based on the positions of the southern components of the jet - where optical depth effects are likely to be less of an issue. Then, knowing the true position of the radio core in the $1.3 \mathrm{GHz}$ map, we can place it onto the emission line image.

Ellipse fitting to the [OIII] data is shown in Figure 8 . As radio emission is detected either side of the nucleus, we have presented the PA of both the northern and southern jets with respect to the radio core, using knots in both the 1.3 and $5 \mathrm{GHz}$ maps. We can see that there is excellent agreement with the radio and [OIII] across a range of radii, but there is significant divergence as the jet extends past the high surface brightness regions and begins to bend. As with PKS 1549-79, we can also see the excellent agreement between the morphologies of the [OIII] continuum and emission line structures.

\section{DISCUSSIONS}

There are several features of these high resolution data that are common to both objects. In the first instance, there are strikingly detailed similarities in the morphologies of the continuum and emission lines detected in the nuclear regions. For example, in both objects the [OIII] continuum images are very similar to the detailed morphologies of the the [OIII] emission in terms of both alignment and scale. This is particularly evident from Figures [6] and 8. Secondly, in both objects the relative alignments in the PAs of the optical morphologies and 
radio knots follow the same pattern; close alignments in the inner regions and increasing disparities at larger radii. In PKS 1549-79 the observed radio core is not exactly aligned with the [OIII] emission, it is offset to the south-east. However, considering the estimated positional accuracy, this offset is not significant. We can see from Figure 6 that the nuclear PAs of both the radio and optical data are consistent. In addition, the PAs fitted to the nuclear isophotes of PKS 1345+12 compare remarkably well with PAs of the radio jet knots for a radius $<0$ '!04 (Figure 8). The isophotes also tentatively follow some of the radio structure, i.e., PAs change in tandem with the jet. In both cases, nevertheless, the optical morphologies do not resemble the nature of the jet on larger scales $\left(>00^{\prime \prime} 15\right)$. This is consistent with the idea that the jets are being deflected at the location of the high surface brightness [OIII] emission.

From inspection of Figures 5 and 7 we find no evidence of bi-conical structures extending beyond the radio jets. The ellipticities of isophotes fitted to the extended emission line data are low $(\sim 0.05)$. On the smallest scales $(\sim 0$.'07) there is also no evidence for bi-cones. Adding this observation to the de-coupling of the radio and optical morphologies at radii greater than $\sim 130$ pc means that we can rule out starburst wind driven outflows; the expected extent of starburst winds (Heckman, Armus \& Miley 1990) are greater than the scales seen here.

The close alignment of PAs between the radio and optical, in the inner regions, provides clear evidence for the relativistic radio jet driven outflow mechanism. This would be consistent with the mechanism powering kiloparsec and halo scale structures. However, the radio structure does extend beyond main [OIII] structure and alignments are not exact on the larger scale. In addition, as mentioned above, the continuum and emission lines show similar structures close to the nucleus. Coincident features such as these may be giving us supplementary clues about the nuclear outflow mechanisms. If we first naively assume that the continuum is dominated by stars and the emission lines are due mostly to gas, then it tells us the gas dynamics are currently consistent with the gravitational potential defined by the stars. However, if the extended nuclear continuum is not stellar, but instead represents AGN-related continuum from the emission line regions, then we may be observing nebular continuum or scattered AGN light. Finally, the nuclear morphologies of the continuum and emission lines may be determined by uneven dust obscuration rather than AGN-related processes. This last case is considerably strong considering the evidence for clear dust lanes in both PKS 1549-79 and PKS 1345+12.

Unfortunately, in the light of this, it is difficult to unambiguously determine whether radiative winds from the AGN, or outflows induced via the radio structures, dominate the driving mechanism. Further factors compound this problem. Even with the high spatial resolution of ACS, there is the fact that the resolution is unfavorable for clearly seeing detailed associations between radio and optical features. The problem is more acute if we consider the possible orientations of the small-scale bi-conical structures indicative of nuclear winds. There is some debate as to the relative alignment to the line of sight for the jet in PKS $1345+12$, however, an orientation close to end on is favored for PKS 1549-79. In that case, the projection of the bi-cone would make resolving the feature especially difficult.

\section{CONCLUSIONS}

We have resolved the emission line outflow regions in two compact radio sources (PKS 1549-79 and PKS $1345+12$ ) using the high resolution channel of ACS. Through morphological comparisons with existing radio data we have investigated the possible driving mechanisms for the observed nuclear outflows. These sources are ideal for this study as they are well known radio galaxies in which nuclear outflows have been unequivocally detected. They also contain all of the possible driving mechanisms (jets, quasars and starbursts).

In both cases we see good agreement with the radio and optical PAs and no evidence for the large scale bi-conical features indicative of starburst driven winds; the emission line morphology shows similar structure and scales to that of the radio. We are thus left with the conclusion that the outflows are most likely not driven by starburst winds. However, these ACS observations are unable to clearly resolve the scales over which a compact nuclear structures may exist, especially if orientations to the line-of-sight are considered. There is also an excellent agreement between the emission line and continuum morphology, which could be attributed to patchy dust obscuration. In addition, while the kinematics do advocate shocks, the ionization mechanism is difficult to couple with shock ionization. Radio jets and radiative AGN winds cannot be unambiguously distinguished as the dominant outflow mechanism.

We extend our thanks to Carlo Stanghellini, Istituto di Radioastronomia del C.N.R, Bologna, Italy, for supplying the $5 \mathrm{GHz}$ radio data for PKS $1345+12$, and to Tasso Tzioumis, CSIRO Radiophysics Laboratory, Pembroke and Vimiera Rds, Marsfield, NSW, Australia, for retrieving the radio data for PKS 1549-79 from Edward King's thesis. We also thank the anonymous referee for their useful comments.

Support for Proposal number HST-GO-09401.10A was provided by NASA through a grant from the Space Telescope Science Institute, which is operated by the Association of Universities for Research in Astronomy, Incorporated, under NASA contract NAS5-26555.

\section{REFERENCES}

Axon, D. J., Capetti, A., Fanti, R., Morganti, R., Robinson, A., \& Spencer, R. 2000, AJ, 120, 2284

Baker, J. C., Hunstead, R. W., Athreya, R. M., Barthel, P. D., de Silva, E., Lehnert, M. D., \& Saunders, R. D. E. 2002, ApJ, 568, 592

Balsara, D. S., \& Krolik, J. H. 1993, ApJ, 402, 109

Bellamy, M. J., Tadhunter, C. N., Morganti, R., Wills, K. A., Holt, J., Taylor, M. D., \& Watson, C. A. 2003, MNRAS, 344, L80
Bicknell, G. V., Dopita, M. A., \& O'Dea, C. P. O. 1997, ApJ, 485 112

Bremer, M. N., Fabian, A. C., \& Crawford, C. S. 1997, MNRAS, 284,213

Carilli, C. L., Perley, R. A., \& Harris, D. E. 1994, MNRAS, 270, 173

Crenshaw, D. M., et al. 2000, AJ, 120, 1731 
Dickson, R., Tadhunter, C., Shaw, M., Clark, N., \& Morganti, R. 1995, MNRAS, 273, L29

Dickson, R. 1997, Ph.D. Thesis, University of Sheffield.

Evans, A. S., Kim, D. C., Mazzarella, J. M., Scoville, N. Z., \& Sanders, D. B. 1999, ApJ, 521, L107

Fabian, A. C., Sanders, J. S., Allen, S. W., Crawford, C. S. Iwasawa, K., Johnstone, R. M., Schmidt, R. W., \& Taylor, G. B. 2003, MNRAS, 344, L43

Ferrarese, L., \& Ford, H. 2005, Space Science Reviews, 116, 523

Gallimore, J. F., Axon, D. J., O'Dea, C. P., Baum, S. A., \& Pedlar, A. 2006, AJ, 132,546

Gilmore, G., \& Shaw, M. A. 1986, Nature, 321, 750

Heckman, T. M., Smith, E. P., Baum, S. A., van Breugel, W. J. M., Miley, G. K., Illingworth, G. D., Bothun, G. D., \& Balick, B. 1986, ApJ, 311, 526

Heckman, T. M., Armus, L., \& Miley, G. K. 1990, ApJS, 74, 833

Holt, J., Tadhunter, C. N., \& Morganti, R. 2003, MNRAS, 342, 227

Holt, J., Tadhunter, C., Morganti, R., Bellamy, M., González Delgado, R. M., Tzioumis, A., \& Inskip, K. J. 2006, MNRAS, 370,1633

Johnston, K. J et al. 1995, AJ, 110, 880

King, E. A. 1994, Ph.D. Thesis, University of Tasmania.

Kinney, A. L., Schmitt, H. R., Clarke, C. J., Pringle, J. E., Ulvestad, J. S., \& Antonucci, R. R. J. 2000, ApJ, 537, 152

Koekemoer, A. M., Fruchter, A. S., Hook, R. N., \& Hack, W. 2002, The 2002 HST Calibration Workshop, Proceedings of a STScI Workshop, 2002, p.339. eds. Arribas, Koekemoer and Whitmore. Krolik, J. H., \& Begelman, M. C. 1986, ApJ, 308, L55

Krongold, Y., Nicastro, F., Brickhouse, N. S., Elvis, M., Liedahl, D. A., \& Mathur, S. 2003, ApJ, 597, 832

Lister, M. L., Kellermann, K. I., Vermeulen, R. C., Cohen, M. H., Zensus, J. A., \& Ros, E. 2003, ApJ, 584, 135

Morganti, R., Oosterloo, T. A., Tadhunter, C. N., van Moorsel, G., Killeen, N., \& Wills, K. A. 2001, MNRAS, 323, 331

Morganti, R., Oosterloo, T. A., Tadhunter, C. N., Vermeulen, R., Pihlström, Y. M., van Moorsel, G., \& Wills, K. A. 2004, A\&A, 424,119

Morganti, R., Tadhunter, C. N., \& Oosterloo, T. A. 2005, A\&A, 444, L9
Nulsen, P. E. J., David, L. P., McNamara, B. R., Jones, C., Forman, W. R., \& Wise, M. 2002, ApJ, 568, 163

O'Dea, C. P. 1998, PASP, 110, 493

O'Dea, C. P., et al. 2002, AJ, 123, 2333

Prestage, R. M., \& Peacock, J. A. 1983, MNRAS, 204, 355

Preston, R. A., et al. 1984, IAU Symp. 110: VLBI and Compact Radio Sources, 110, 67

Rodriguez Zaurin, J., Holt, J., Tadhunter, C. N., \& Gonzalez Delgado, R. M. 2006, ArXiv Astrophysics e-prints, arXiv:astro-ph/0612578

Roy, A. L., \& Norris, R. P. 1997, MNRAS, 289, 824

Scoville, N. Z., et al. 2000, AJ, 119, 991

Silk, J., \& Rees, M. J. 1998, A\&A, 331, L1

Stanghellini, C., O'Dea, C. P., Baum, S. A., \& Laurikainen, E. 1993, ApJS, 88, 1

Stanghellini, C., O'Dea, C. P., Baum, S. A., Dallacasa, D., Fanti, R., \& Fanti, C. 1997, A\&A, 325, 943

Stanghellini, C., Dallacasa, D., O'Dea, C. P., Baum, S. A., Fanti, R., \& Fanti, C. 2001, A\&A, 377, 377

Stanghellini, C., O'Dea, C. P., Dallacasa, D., Cassaro, P., Baum, S. A., Fanti, R., \& Fanti, C. 2005, A\&A, 443, 891

Tadhunter, C. N., Scarrott, S. M., Draper, P., \& Rolph, C. 1992, MNRAS, 256, 53P

Tadhunter, C. N., Packham, C., Axon, D. J., Jackson, N. J., Hough, J. H., Robinson, A., Young, S., \& Sparks, W. 1999, ApJ, 512, L91

Tadhunter, C., Wills, K., Morganti, R., Oosterloo, T., \& Dickson, R. 2001, MNRAS, 327, 227

Tadhunter, C., Robinson, T. G., González Delgado, R. M., Wills, K., \& Morganti, R. 2005, MNRAS, 356, 480

Veilleux, S. 2004, Astronomical Society of the Pacific Conference Series, 320, 277, eds. Aalto et al.

Wyithe, J. S. B., \& Loeb, A. 2003, ApJ, 595, 614

Xiang, L., Stanghellini, C., Dallacasa, D., \& Haiyan, Z. 2002, A\&A, 385,768 